\title{
RESPONSABILIDAD SOCIAL Y TRANSPARENCIA A TRAVÉS DE LA WEB: UN ANÁLISIS APLICADO A LAS COOPERATIVAS AGROALIMENTARIAS ESPAÑOLAS
}

\author{
POR \\ Ricardo MUÑOZ PASADAS ${ }^{1}$ y \\ Pilar TIRADO VALENCIA ${ }^{2}$
}

\section{RESUMEN}

Las sociedades cooperativas han aplicado desde sus orígenes los valores y los principios que están implícitos en el concepto de la Responsabilidad Social Empresarial (RSE). El objetivo de este trabajo es analizar la relación entre la responsabilidad social, el gobierno corporativo y la transparencia en las sociedades cooperativas agroalimentarias españolas, dada la vinculación existente entre los principios inspiradores de la cultura cooperativa y la RSE, y de esta última con los niveles de divulgación de información. El trabajo analiza las características de la información sobre RSE que estas empresas difunden a través de sus páginas webs, basándose en los contenidos de la iniciativa Global Reporting Initiative, e intenta demostrar si existen diferencias significativas entre los niveles alcanzados por las cooperativas de primer grado y las de grado ulterior. Finalmente se analiza la relación entre los niveles de difusión de información y el tamaño de la cooperativa o sus años de antigüedad.

Palabras clave: Transparencia, Responsabilidad Social Empresarial (RSE), Gobierno Corporativo, Global Reporting Initiative (GRI), Cooperativas Agroalimentarias.

Claves Econlit: G300, M140, P130

\footnotetext{
${ }^{1}$ Dirección de correo electrónico: rmunozp@ covap.es

${ }^{2}$ Universidad Loyola Andalucía. Dirección de correo electrónico: ptirado@etea.com

REVESCO No 114 - Primer Cuatrimestre 2014 - ISSN: 1885-8031 - www.ucm.es/info/revesco

http://dx.doi.org/10.5209/rev_REVE.2014.v114.44293

Fecha de recepción: 11/04/2013

Fecha de aceptación: 29/10/2013
} 


\title{
SOCIAL RESPONSIBILITY AND TRANSPARENCY IN THE WEB: AN ANALYSIS APPLIED TO SPANISH AGRO-FOOD COOPERATIVES
}

\begin{abstract}
The values and principles of Corporate Social Responsibility (CSR) have been applied by Cooperative societies since their origins. Given the link of CSR with cooperative culture principles and information spreading, the aim of this paper is to analyze the relationship between social responsibility, corporate governance and transparency in the Spanish agrofood cooperatives. This paper analyzes the CSR information these companies spreads across the web, according the Global Reporting Initiative contents. The paper tries to show whether there are significant differences between the levels reached by primary and secondary or tertiary cooperatives. Finally, the relationship between levels of information dissemination and cooperatives size or age is analyzed.
\end{abstract}

Keywords: Transparency, Corporate Social Responsibility (CSR), Corporate Governance, Global Reporting Initiative (GRI), Agro-food Cooperatives.

\section{INTRODUCCIÓN}

En el año 2007, la Alianza Cooperativa Internacional (ACI), con motivo del $85^{\circ}$ Día Internacional de la Cooperación y en el $13^{\circ}$ Aniversario del día Mundial de las Cooperativas establecido por Naciones Unidas, difundió, como lo hace anualmente, su tradicional mensaje, que en esta ocasión llevaba por título "Los Principios y Valores Cooperativos para la Responsabilidad Social de las Empresas", afirmando entre otras cuestiones que "se celebra la contribución que los cooperativistas han hecho y continúan haciendo como ciudadanos responsables en las cooperativas de todo el mundo (...). La Responsabilidad Social Empresarial (RSE) intenta poner el énfasis en demostrar cómo el mundo de los negocios puede mejorar la sociedad a través de comportamientos e informes transparentes e íntegros (...)".

De muchas formas distintas, el movimiento cooperativo ha sido pionero en el desarrollo y la práctica de la RSE desde hace más de 150 años. Las sociedades cooperativas han aplicado desde sus orígenes los valores y los principios que están implícitos en el propio concepto de la RSE (Carrasco, 2005; Server y Capó, 2009; Mozas y Puentes, 2010; Horrach y Socias, 2011). Es más, las entidades de la Economía Social (sociedades cooperativas, 
sociedades laborales, etc.) presentan una cierta ventaja respecto a las empresas mercantiles debido a que ya cuentan con una ideología propia de carácter solidaria, participativa, igualitaria y democrática que está en consonancia con los compromisos que las empresas adquieren en las relaciones con sus grupos de interés en la puesta en práctica de la RSE (Olmedo y otros, 2012).

Por su parte, las cooperativas agroalimentarias son responsables desde un punto de vista económico, por crear riqueza a la vez que empleo estable y duradero, siendo el motor económico de algunas zonas rurales especialmente deprimidas. Son igualmente responsables económicamente por defender cada uno de los micro negocios o explotaciones familiares de sus socios y por contribuir de una manera determinante a su viabilidad. En países en vías de desarrollo desempeñan un papel esencial en el impulso de sus economías. Las cooperativas son responsables socialmente por contribuir al bienestar de sus miembros y de las comunidades locales donde residen, por ser solidarias y democráticas, por dar prioridad a las personas sobre el capital, por sus políticas de participación igualitaria, o por su trabajo con colectivos especialmente desfavorecidos, entre otras cuestiones. Son igualmente responsables porque trabajan en favor de todos sus grupos de interés, siendo el socio pieza angular por sus diferentes roles en la actividad cooperativa (Arcas y otros, 2011). Lo son también ambientalmente, por su defensa del entorno natural de las zonas en las que se ubican y por el mantenimiento de la cultura y las tradiciones.

Las cooperativas no sólo han sido un precedente en el ámbito de la RSE, sino que están llamadas a jugar un papel fundamental y de equilibrio en el futuro modelo productivo que está por llegar (Server y Capó, 2009).

Por su parte, las cooperativas agroalimentarias se convierten en un elemento clave para el desarrollo del sector, por su importancia social y económica. Según el dictamen del Comité Económico y Social Europeo (2012) sobre "Cooperativas y Desarrollo agroalimentario" (...) "En la búsqueda de un modelo económico más sostenible, el cooperativismo se erige como una alternativa competitiva y eficiente, que ofrece nuevas respuestas a los desequilibrios de la cadena de valor del sector agroalimentario y que, a su vez, promueve el empleo y fomenta las cadenas alimentarias locales, la seguridad alimentaria, la participación y la responsabilidad social”. En un mundo cada vez más globalizado y cuando las ayudas al productor europeo vienen reduciéndose en los últimos años, las nuevas tendencias de la Política Agraria Comunitaria se centran en aspectos como la 
multifuncionalidad y el desarrollo rural, surgiendo nuevos desafíos para el sector agrario y para el socio cooperativo español y europeo. Las cooperativas agroalimentarias deben ser organizaciones que lideren estas nuevas preocupaciones.

La vinculación entre los principios cooperativos y los valores que el cooperativismo promueve, y el compromiso inherente en el propio concepto de la RSE, justifican la necesidad de que las cooperativas articulen mecanismos en sus procesos de gestión para canalizar el conjunto de sus esfuerzos en favor de una mayor responsabilidad social. Serán necesarias políticas, planes estratégicos, iniciativas concretas, una estructura formal que apoye este proceso de gestión, mecanismos para la evaluación y el control de las variables de la RSE, canales de comunicación, etc. Acciones que tienen por finalidad dar respuesta a las expectativas de sus grupos de interés, mejorar la transparencia, la reputación y la legitimidad de las cooperativas.

El objetivo de este trabajo es doble. Por un lado, analizar los niveles de difusión de información sobre RSE de las cooperativas agroalimentarias españolas a través de sus páginas webs, y determinar las características más relevantes de sus contenidos mediante el estudio de las variables de RSE que están presentes en internet, con la finalidad de identificar pautas y perfiles de la información suministrada que puedan ser relevantes en sus políticas de RSE de cara a una mayor transparencia. Por otro, contrastar si determinadas características como el tamaño y la antigüedad, pudieran estar influyendo en los niveles de divulgación de información sobre responsabilidad social, bajo la hipótesis de que al disponer las cooperativas de mayor tamaño de mayores recursos para aplicar en sus políticas de comunicación y diálogo con sus grupos de interés, y al haber alcanzado las cooperativas de mayor edad una mayor madurez en cuanto a la asunción de la responsabilidad social y a la transparencia, los niveles de difusión de información podrían resultar mayores.

Tras esta introducción, en el segundo apartado se justifica la necesidad del estudio en el que se relacionan el cooperativismo con la responsabilidad social, con el buen gobierno y con la necesidad de unos niveles adecuados de transparencia mediante el uso de la web. En el tercer apartado se describen las variables de RSE analizadas y la metodología empleada sobre una muestra de 39 grandes cooperativas agroalimentarias españolas. En el apartado cuatro se presentan los resultados obtenidos acerca de los niveles de difusión de información sobre RSE en la web de las cooperativas de la muestra, tras analizar un total de 32 variables que podrían 
estar influyendo en dichos niveles. Finalmente en el apartado cinco se presentan las principales conclusiones y las limitaciones del estudio.

\section{JUSTIFICACIÓN DEL ESTUDIO}

El gobierno corporativo se ha convertido en un aspecto clave para una gestión eficiente y de la responsabilidad social de las cooperativas (Puentes y otros, 2009) cuando es concebido desde una óptica pluralista que comprende la gestión de las relaciones con todos los grupos de interés (Pedrosa y García, 2011). Los enfoques de gobierno corporativo y responsabilidad social están íntimamente ligados y tienen una influencia directa y positiva sobre la reputación (Olmedo y otros, 2012). El objeto social de las cooperativas y su gestión democrática hace que sean más propensas a fijar criterios de buen gobierno, transparencia y responsabilidad social, superando al resto de sociedades capitalistas en general (Palomo y Valor, 2004).

El buen gobierno cooperativo habrá de estar dirigido tanto hacia la participación democrática de los socios en las actividades de la cooperativa como hacia el correcto funcionamiento de los órganos de gobierno y control, así como hacia una gestión sostenible que satisfaga las expectativas de todos sus grupos de interés (Salazar, 2010). Desde esta perspectiva, el modelo de gobierno corporativo se ha de dirigir hacia la gestión de las relaciones con todos los stakeholders.

El buen gobierno de la cooperativa exige que se identifiquen los grupos de interés prioritarios, en términos de poder de control, legitimidad moral o contractual y urgencia de sus demandas; que se incorporen las expectativas de los grupos en las políticas, y que se evalúe si se da respuesta a sus demandas, con el objeto de lograr un alineamiento de intereses entre los grupos y la propia sociedad cooperativa (Pedrosa y García, 2011) evitando las asimetrías de información entre los partícipes.

Para ello, uno de los requerimientos de la RSE y del buen gobierno es la transparencia, es decir, la comunicación de los compromisos adquiridos con los grupos de interés, de los impactos económicos, sociales y medioambientales de sus actuaciones; y de la estructura organizativa y operativa de la entidad (Horrach y Socias, 2011). La transparencia y la rendición de cuentas son prácticas esenciales del buen gobierno, entendiendo por estas no sólo las que afectan directamente al funcionamiento del órgano de gobierno, sino todas aquellas 
que contribuyen a fortalecer el capital institucional de la organización, clave para su sostenibilidad (Rey y Martín, 2011).

La transparencia es un medio en tanto en cuanto a través de ella las empresas pueden alcanzar legitimidad; pero también es un fin de las organizaciones en sí mismas, porque genera confianza e incide en su reputación, además de acrecentar la fidelización de los socios (Morales, 2005). La transparencia supone un ejercicio de responsabilidad, pero al mismo tiempo es un medio para que los compromisos asumidos por la empresa sean conocidos y aprobados por sus grupos de interés. En el caso de las cooperativas, puede incluso instrumentalizarse como un mecanismo de control externo que justifique los niveles de apoyo económico, financiero y fiscal alcanzados a nivel estatal y autonómico (Morales y Ariza, 2010).

Gobierno corporativo, rendición de cuentas, transparencia, comunicación y evaluación son cuestiones que están íntimamente relacionadas, en la medida que la transparencia provee de un marco que permite mejorar la información disponible para los stakeholders y dotar a la entidad de una mayor credibilidad social (Muñoz y otros, 2010). Sin embargo, algunos estudios demuestran que en el ámbito cooperativo la información se dirige fundamentalmente a ciertos grupos de interés dominantes con fines utilitaristas, bien para que aprueben la gestión del órgano de dirección o bien para justificar los recursos recibidos de las administraciones públicas o para poder acceder a ellos en el futuro (Horrach y Socias, 2011).

Las principales motivaciones a la hora de divulgar la información sobre RSE están relacionadas con la responsabilidad hacia los grupos de interés y la legitimidad que aporta a la actividad de la cooperativa. A pesar de la relevancia de la cuestión, algunas cooperativas siguen viendo algunos inconvenientes en la difusión de información relativos al coste de la emisión de información, la inercia de la entidad, o el desconocimiento y la dificultad en la medición de las cuestiones sociales. Algunas otras evalúan el riesgo reputacional que corren ante una interpretación negativa de la información (Horrach y Socias, 2011).

La creciente preocupación en relación con la transparencia ha llevado a la publicación de directrices internacionales sobre la difusión de las prácticas sobre RSE de forma generalizada. Muchas de estas directrices se han venido formalizando a través de diferentes propuestas normalizadoras de las políticas para la gestión de la responsabilidad social y de los contenidos de la información para la sostenibilidad. Entre las guías más relevantes se pueden 
mencionar la de la OCDE (2006), el Banco Mundial (2007), Accountability (2008a, 2008b) y la Global Compact de Naciones Unidas (2009); pero la iniciativa de mayor difusión en el ámbito de la emisión de información de carácter voluntario hasta el momento es la elaborada por la organización Global Reporting Initiative (GRI) (2011a) cuya tercera versión revisada G3.1 se publicó en 2011.

Por otra parte, el uso de internet puede constituir un medio adecuado para el establecimiento de canales de comunicación y diálogo con los grupos de interés, así como para la difusión de información sobre la responsabilidad social. Sin embargo, el nivel de presencia en la web por parte de las cooperativas es aún escaso debido a que no están claras sus ventajas y a la percepción de una gran complejidad en su mantenimiento y uso (López y otros, 2012). En el caso de las cooperativas valencianas, en el año 2005 sólo un 26,6\% de las empresas contaba con su propia página web, mientras que el nivel internacional de presencia llega a ser del 75\% (Ferrer y otros, 2007). Como cabría esperar, la ausencia de web es más acusada entre las cooperativas de menor tamaño. Para Juliá y otros (2004), en general, las cooperativas de todos los sectores que utilizan el dominio "coop" no aprovechan las oportunidades que ofrece la tecnología para atender, entre otros, al quinto principio cooperativo sobre "Educación, formación e información", con especial incidencia en la cuestión de la información. Igualmente, el estudio de Mozas y otros (2010), pone de manifiesto que en el caso de las cooperativas andaluzas, la utilización de la web como medio de comunicación fundamental de la información referente a RSE, se puede considerar deficiente.

En el caso concreto de las cooperativas agrarias, el informe del Observatorio Socioeconómico del Cooperativismo Agrario Español (2011) concluye que en el año 2011 tan sólo el 53,3\% de las empresas cooperativas agrarias españolas tenían página web propia. En concreto, según el estudio de Montegut y otros (2013) sólo un 55,1\% de las cooperativas agrarias de la provincia de Lleida afirma disponer de un sitio web propio y la frecuencia de actualización de contenidos es bastante esporádica. Las cooperativas agroalimentarias no deben quedarse al margen de la presencia en internet, para aprovechar todas las ventajas que ofrecen las nuevas tecnologías de la información (Montegut y otros, 2013), además de compatibilizarla con sus propios principios (Juliá y otros, 2004; Rey y Martín, 2011) y mejorar la transparencia. 
Las dificultades a la hora de identificar qué información es relevante y satisface las expectativas de los grupos de interés, y el uso incipiente de internet como medio para la gestión de las relaciones de las cooperativas con sus stakeholders en el ámbito de la RSE, a pesar de tratarse de un medio adecuado para la transparencia y el buen gobierno, justifican la necesidad de este estudio en el que se analizan las variables de responsabilidad social presentes en las webs de las cooperativas agroalimentarias españolas, así como los factores que podrían explicar los distintos niveles de difusión de información a través de este medio.

\section{ANÁLISIS EMPÍRICO}

\subsection{Definición de variables}

No existe una propuesta consensuada sobre las variables representativas de la RSE que deban ser difundidas a efectos de transparencia a nivel sectorial en el ámbito cooperativo agroalimentario, ni incluso relacionada con el gobierno corporativo. Son numerosos los estudios que se han ocupado de la identificación de variables que permitan hacer una evaluación del desempeño en términos de responsabilidad social de las entidades de la Economía Social, dando lugar a diferentes propuestas (Pérez y Gargallo, 2005; Arcas y Briones, 2009; Pérez y otros, 2009; Mozas y otros, 2010; Castilla y Gallardo, 2011; Degli y Portale, 2011; Horrach y Socias, 2011; Rey y Martín, 2011).

Para la realización del estudio sobre los niveles de transparencia ofrecidos por las empresas a través de sus páginas webs en términos de RSE y, particularmente, en cuanto al gobierno corporativo, se ha diseñado un cuestionario a partir de la selección de un conjunto de variables basadas en la Guía para la elaboración de Memorias de Sostenibilidad del organismo internacional Global Reporting Initiative (GRI) (2011a). Sin lugar a dudas se trata de la iniciativa con un mayor reconocimiento a nivel internacional en el ámbito de la normalización de contenidos de la información relativos a la RSE.

Para el análisis efectuado sobre la transparencia en materia de gobierno corporativo, el estudio se ha limitado a la primera parte del documento, donde se incluyen los aspectos generales de la entidad, su estrategia, las relaciones con los grupos de interés, el gobierno y los parámetros de la información divulgada, entre otros. Todas estas cuestiones se han agrupado en cinco categorías, coincidentes con los cuatro primeros apartados del documento GRI (2011a), dividiéndose el último apartado, a su vez, en dos. Las categorías que se analizan en el estudio son las siguientes: 
A) Estrategia y análisis (categoría 1). La primera categoría incide en la estrategia de la compañía a través de la declaración personal del presidente de la entidad, principalmente en materia de sostenibilidad y si fuese así, sobre los asuntos prioritarios recogidos en el informe de sostenibilidad.

B) Perfil de la organización (categoría 2). Para la segunda categoría del cuestionario, la que hace referencia a la entidad propiamente dicha, se examina si las empresas informan en su web de cuestiones sobre la propia organización, tales como productos, participación en otras compañías y estructura societaria, localización de la sede, países donde produce, donde opera, forma jurídica, mercados o su dimensión medida a través de distintas magnitudes, así como de la historia de la entidad o de los premios recibidos en términos de sostenibilidad.

C) Parámetros de la información (categoría 3). El tercero de los bloques examinados se refiere a los parámetros de la información transmitida respecto a la sostenibilidad, haciendo referencia al periodo cubierto por la información, la existencia de un punto de contacto, el alcance y cobertura de la información, así como la existencia de limitaciones en los contenidos divulgados y la existencia de procesos de verificación externa. En el caso de no hacer referencia a estos aspectos, se pregunta si se menciona cómo se resolverán en un futuro estas cuestiones.

D) Gobierno Corporativo (categoría 4). El gobierno corporativo ocupa la cuarta de las categorías. En las mismas materias, social, ambiental y económica, se pregunta si se informa de las responsabilidades del consejo y del presidente, en concreto, si estos aspectos se asocian con la retribución. Internamente, se plantea si se informa de los mecanismos establecidos para evitar conflictos de intereses. De manera global, se pregunta por la definición de la misión, de una declaración de principios o de valores de la entidad, o por la existencia de códigos de conducta así como el estado en que se encuentra su implementación. Por otro lado, se examina si el consejo ejerce funciones concretas de supervisión ante cuestiones que afecten a la sostenibilidad y, recíprocamente, si existen mecanismos que controlen la gestión del consejo en este sentido.

E) Grupos de interés (categoría 5). Por último, la quinta categoría del cuestionario se centra en los grupos de interés. Primero se analiza si se trabaja en su identificación y selección, si existen canales para la identificación de sus demandas y de los asuntos 
materiales de especial relevancia, y se pregunta si existen mecanismos de retroalimentación que garanticen el aprendizaje. Se analiza el grado de compromiso con los grupos, si existen procedimientos para hacer frente a sus quejas, si se evalúa periódicamente la gestión, si se le comunican los resultados de esta evaluación, o si se materializan procesos concretos para la coordinación de las iniciativas para satisfacer sus expectativas.

Para el estudio de los niveles de difusión de información de las cooperativas agroalimentarias españolas en cada una de estas cinco categorías se han incorporado al trabajo un total de 32 variables. Para la selección de las variables se han considerado todos los aspectos englobados en GRI (2011a) en cada una de las categorías descritas. Este trabajo se ha complementado con la incorporación de otras variables procedentes de los documentos sobre adaptaciones sectoriales para el sector de entidades no lucrativas Non Government Sector Supplement NGOSS (GRI, 2011b) y del suplemento elaborado también recientemente para el sector de la Industria de la Alimentación, Food Processing Sector Supplement (FPSS) (GRI, 2011c). Tras un estudio de la totalidad de los apartados desarrollados en la Guía y en los dos suplementos se han descartado aquellas variables menos relevantes desde el punto de vista de las cooperativas y del sector objeto de estudio. Así, la relevancia y practicidad de la información es el criterio escogido para la selección de las 32 variables incorporadas al cuestionario, agrupadas en las cinco categorías descritas.

\subsection{Selección de la muestra analizada}

La muestra objeto de estudio está formada por las 39 empresas cooperativas agroalimentarias españolas de mayor tamaño según la información disponible en el listado de la Confederación Empresarial Española de la Economía Social (CEPES) ${ }^{3}$. Se trata de aquellas cooperativas agroalimentarias cuyo volumen de facturación superó los 50 millones de $€$ en el ejercicio 2010. La elección de las cooperativas de la muestra en función de su volumen de ingresos responde a dos razones. En primer lugar, siendo el objetivo de este trabajo el análisis de la información sobre RSE y gobierno corporativo divulgada por este tipo de organizaciones en internet, el hecho de que dispongan de página web es un requisito indispensable ya que ésta constituye la fuente de obtención de datos sobre las variables analizadas. Las empresas más grandes disponen de mayores recursos y por lo tanto existen mayores posibilidades de que dispongan de página web propia y del personal necesario para

\footnotetext{
${ }^{3}$ http://www.cepes.es/Ranking
} 
el mantenimiento de unos contenidos más amplios y actualizados. En segundo lugar, se intenta evitar una excesiva atomización de la muestra.

De las 39 grandes cooperativas agroalimentarias españolas que constituyen la muestra, 20 son de primer grado y 19 de segundo o ulterior grado. El tamaño reducido de la muestra conlleva a que el estudio empírico se platee tan sólo con un carácter exploratorio.

\subsection{Metodología}

La asignación de valores a cada una de las 32 variables sobre gobierno corporativo y RSE identificadas en el estudio, se realiza a través de una ponderación numérica, otorgando cuatro tipos de puntuaciones a cada una de las variables en función de la cantidad y de la calidad de la información disponible en la web, siguiendo la metodología aplicada por Mozas y otros (2010):

A) No se informa/no aparece (valoración asignada 0). La primera opción implica la ausencia total de información sobre la variable analizada, poniendo de manifiesto una falta de transparencia sobre el asunto en cuestión.

B) Se declara su existencia (valoración asignada 1). La segunda opción da muestras de una escasa preocupación por la variable de cara a los grupos de interés, ya que tan sólo se plasma de una manera sucinta en la web.

C) Información genérica (valoración asignada 2). Con esta valoración se puede pensar que la entidad concede una cierta relevancia a la cuestión, pero no se informa de una manera pormenorizada por falta de capacidad o de recursos.

D) Información completa (valoración asignada 3). Se constata la importancia que la entidad concede a la variable analizada, formando parte de manera muy completa y exhaustiva de la información que se comunica a los grupos de interés a través de la web.

Las puntuaciones asignadas (de 0 a 3) permiten aplicar al estudio diferentes metodologías y obtener distintos tipos de resultados.

1. Análisis de frecuencias de cada una de las variables. Este análisis puede ser relevante a la hora de identificar qué aspectos concretos son objeto de unos mayores índices de transparencia, y cuáles son las cuestiones que presentan unas mayores carencias de 
información. De esta forma se podrían hacer propuestas de mejora en los contenidos de la información divulgada a través de las páginas webs como parte de las políticas de RSE de las cooperativas agroalimentarias. La frecuencia de cada una de las variables es un porcentaje que se ha calculado dividiendo la suma de todas las puntuaciones asignadas a las mismas tras consultar las páginas webs de las 39 cooperativas agroalimentarias de la muestra, entre un total de 117 puntos que es el máximo de puntos posibles: asignación de 3 puntos a la variable, en las 39 cooperativas analizadas.

2. Análisis de frecuencias para cada categoría, es decir, para cada uno de los cinco bloques en los que se agrupan las variables del estudio, y en términos generales. Los resultados obtenidos podrían poner de manifiesto si se está dando respuesta a todos los contenidos relativos a la comunicación en términos de sostenibilidad. La frecuencia de cada categoría es la media ponderada de las frecuencias de todas las variables incluidas en ella. Asimismo, se ha calculado la desviación típica de cada categoría para determinar el grado de dispersión de la información. Finalmente, en este análisis se han discriminado los resultados en función del grado de la cooperativa para tratar de determinar si existen unos mayores niveles de desempeño en cuanto a los niveles de información mediante la realización de una prueba no paramétrica.

3. Análisis de la correlación de las frecuencias de las categorías con el tamaño de la cooperativa, medido por medio de tres indicadores: la facturación, el número de empleos directos y el número de socios de base. Igualmente se han correlacionado las frecuencias de las categorías con los años de antigüedad de la cooperativa para intentar demostrar si existe alguna dependencia de los niveles de información con respecto a la mayor trayectoria histórica de la cooperativa y su mayor grado de madurez en el tiempo. Estos resultados pueden servir para concluir si el compromiso con la RSE en cuanto a transparencia y comunicación con los grupos de interés, se ve influido por la disponibilidad de recursos, y si está relacionada con la madurez de la cooperativa, puesto que es necesario un cierto tiempo para interiorizarse. 


\section{RESULTADOS}

\subsection{Análisis de las variables de la Responsabilidad Social}

Los resultados obtenidos sobre las frecuencias para cada una de las 32 variables del estudio se resumen en la tabla 1 , junto con su media y su desviación típica.

Tabla 1. Frecuencias de las variables

\begin{tabular}{|c|c|c|c|c|}
\hline & & Frecuencia & Media & Desv.Tip. \\
\hline & CATEGORÍA 1. ESTRATEGIA Y ANÁLISIS & & & \\
\hline 1 & $\begin{array}{l}\text { Existe una declaración del máximo responsable sobre la relevancia } \\
\text { de la sostenibilidad para la organización }\end{array}$ & $21 \%$ & 0,62 & 1,11 \\
\hline 2 & $\begin{array}{l}\text { Prioridades estratégicas y asuntos clave sobre sostenibilidad en el } \\
\text { medio/corto plazo y su relación con el largo }\end{array}$ & $13 \%$ & 0,38 & 0,85 \\
\hline 3 & Los principales eventos, logros y fracasos registrados & $6 \%$ & 0,18 & 0,51 \\
\hline 4 & Perspectivas de grado de cumplimiento en relación a los objetivos & $2 \%$ & 0,05 & 0,32 \\
\hline 5 & $\begin{array}{l}\text { Descripción de los principales impactos, riesgos y oportunidades } \\
\text { para la entidad, y sus efectos sobre los grupos de interés }\end{array}$ & $6 \%$ & 0,18 & 0,51 \\
\hline \multirow[t]{2}{*}{6} & $\begin{array}{l}\text { El impacto en las perspectivas a largo plazo y en el desempeño } \\
\text { financiero de la organización }\end{array}$ & $3 \%$ & 0,10 & 0,38 \\
\hline & CATEGORÍA 2. PERFIL DE LA ORGANIZACIÓN & & & \\
\hline 7 & $\begin{array}{l}\text { Se identifican comercialmente sus productos en el mercado } \\
\text { mediante marca propia o empresa comercializadora }\end{array}$ & $77 \%$ & 2,31 & 1,13 \\
\hline 8 & Se identifican áreas de responsabilidad y empresas participadas & $32 \%$ & 0,95 & 1,05 \\
\hline 9 & $\begin{array}{l}\text { Se identifica la localización de la sede principal de la entidad y de } \\
\text { sus centros de producción }\end{array}$ & $69 \%$ & 2,08 & 1,13 \\
\hline 10 & $\begin{array}{l}\text { Se identifican los países en los que se opera, y se citan los más } \\
\text { relevantes en aspectos de sostenibilidad }\end{array}$ & $26 \%$ & 0,77 & 1,09 \\
\hline 11 & Se indica la forma jurídica de la entidad & $79 \%$ & 2,38 & 1,23 \\
\hline 12 & Mercados servidos (por países, sectores y tipos de clientes) & $17 \%$ & 0,51 & 0,79 \\
\hline 13 & $\begin{array}{l}\text { Dimensión (empleados, ventas, deuda, cantidad de producto) y } \\
\text { otros (activo, propietarios, desglose por país/región) }\end{array}$ & $32 \%$ & 0,95 & 0,83 \\
\hline 14 & $\begin{array}{l}\text { Informa sobre los cambios relevantes acontecidos a lo largo de la } \\
\text { historia de la entidad }\end{array}$ & $35 \%$ & 1,05 & 1,12 \\
\hline \multirow[t]{2}{*}{15} & Informa de los premios o distinciones obtenidos & $35 \%$ & 1,05 & 1,23 \\
\hline & CATEGORÍA 3. PARÁMETROS DE LA INFORMACIÓN & & & \\
\hline 16 & $\begin{array}{l}\text { Se identifica la fecha del último informe de RSE o Sostenibilidad } \\
\text { publicado (si lo hay) }\end{array}$ & $8 \%$ & 0,23 & 0,81 \\
\hline 17 & $\begin{array}{l}\text { Existe punto de contacto general para cuestiones relativas a la } \\
\text { información suministrada }\end{array}$ & $87 \%$ & 2,62 & 1,02 \\
\hline 18 & $\begin{array}{l}\text { Define los contenidos económico, social y medioambiental, } \\
\text { prioriza aspectos e identifica sus grupos de interés de manera clara }\end{array}$ & $17 \%$ & 0,51 & 0,94 \\
\hline \multirow[t]{2}{*}{19} & $\begin{array}{l}\text { Si no se cubren todos los impactos económicos, sociales y } \\
\text { ambientales, se indica cómo se alcanzarán }\end{array}$ & $10 \%$ & 0,31 & 0,77 \\
\hline & CATEGORÍA 4. GOBIERNO CORPORATIVO & & & \\
\hline 20 & $\begin{array}{l}\text { Composición del Consejo y responsabilidades asignadas en } \\
\text { materia económica, social y medioambiental }\end{array}$ & $10 \%$ & 0,31 & 0,77 \\
\hline 21 & $\begin{array}{l}\text { Se informa de si el presidente ostenta cargo ejecutivo y de sus } \\
\text { funciones }\end{array}$ & $11 \%$ & 0,33 & 0,87 \\
\hline 22 & $\begin{array}{l}\text { Existe relación entre retribución y resultado económico, social y } \\
\text { ambiental de los miembros del Consejo, altos directivos y } \\
\text { ejecutivos (incluidos los acuerdos de abandono) }\end{array}$ & $3 \%$ & 0,08 & 0,48 \\
\hline 23 & Existen procedimientos para evitar conflictos de intereses en el & $5 \%$ & 0,15 & 0,67 \\
\hline
\end{tabular}




\begin{tabular}{|c|c|c|c|c|}
\hline & máximo órgano de gobierno & & & \\
\hline 24 & $\begin{array}{l}\text { Misión y valores, códigos de conducta, principios relevantes en } \\
\text { relación a la sostenibilidad, y estado de su implementación } \\
\text { (también por regiones y departamentos, y si hacen referencia a } \\
\text { estándares internacionales). }\end{array}$ & $17 \%$ & 0,51 & 0,85 \\
\hline 25 & $\begin{array}{l}\text { Procedimientos del Consejo para supervisar la sostenibilidad en la } \\
\text { organización, los riesgos y oportunidades, y el cumplimiento de } \\
\text { estándares internacionales }\end{array}$ & $6 \%$ & 0,18 & 0,68 \\
\hline \multirow[t]{2}{*}{26} & $\begin{array}{l}\text { Procedimientos para evaluar al Consejo, en especial con respecto } \\
\text { al desempeño económico, ambiental y social }\end{array}$ & $0 \%$ & 0,00 & 0,00 \\
\hline & CATEGORÍA 5. GRUPOS DE INTERÉS & & & \\
\hline 27 & $\begin{array}{l}\text { Define las bases para la identificación y selección de grupos de } \\
\text { interés con los que la organización se compromete }\end{array}$ & $12 \%$ & 0,36 & 0,74 \\
\hline 28 & $\begin{array}{l}\text { Aspectos de interés que han surgido a través de la participación de } \\
\text { los grupos de interés, y la forma en la que ha respondido la } \\
\text { organización }\end{array}$ & $5 \%$ & 0,15 & 0,54 \\
\hline 29 & $\begin{array}{l}\text { NGO1 Compromiso con las partes interesadas mediante procesos } \\
\text { para la participación de los grupos de interés afectados en el } \\
\text { diseño, implementación, monitoreo y evaluación de políticas y } \\
\text { programas }\end{array}$ & $10 \%$ & 0,31 & 0,61 \\
\hline 30 & $\begin{array}{l}\text { NGO2 Comentarios, quejas y mecanismos de retroalimentación en } \\
\text { relación con el no cumplimiento de los programas y políticas } \\
\text { diseñadas con los grupos de interés }\end{array}$ & $4 \%$ & 0,13 & 0,47 \\
\hline 31 & $\begin{array}{l}\text { NGO3 Existen sistemas de medición (incluida su eficacia), } \\
\text { evaluación y aprendizaje, y comunicación de resultados a los } \\
\text { grupos de interés }\end{array}$ & $5 \%$ & 0,15 & 0,54 \\
\hline 32 & $\begin{array}{l}\text { NGO6 Establecimiento de procesos para la coordinación de las } \\
\text { iniciativas para satisfacer las expectativas de los diferentes grupos } \\
\text { de interés }\end{array}$ & $15 \%$ & 0,46 & 0,88 \\
\hline
\end{tabular}

Fuente: Elaboración propia.

Los resultados de la tabla 1 ponen de manifiesto unos escasos niveles de difusión de información sobre RSE ya que tan sólo cuatro variables destacan sobre las demás con porcentajes que superan el $50 \%$ de la frecuencia; estas variables son:

- La variable 17 (87\%) relacionada con la existencia de un punto de contacto en la web tiene un alto índice de presencia. Sin embargo, no se ha podido constatar la calidad de las respuestas ofrecidas a los grupos de interés por este medio, medida en tiempo y satisfacción con solución de la cuestión planteada.

- Según la variable 11 (79\%) se suele indicar la forma jurídica de la entidad y se identifica claramente como empresa de naturaleza cooperativa.

- La variable 7 (77\%) pone de manifiesto que se identifican y se describen en la web los productos comercializados por la cooperativa mediante sus marcas.

- Como revela la variable 9 (69\%) en las páginas webs analizadas se suele identificar la sede de la organización y los principales centros de producción. 
Un análisis de la naturaleza de las cuestiones que aparecen con mayor frecuencia revela que éstas están relacionadas fundamentalmente con la identificación de la organización y de su actividad principal, sin embargo son cuestiones que tienen una escasa materialidad de cara a la responsabilidad social de la empresa. Se trata de aspectos que no desvelan la existencia de políticas sobre RSE, ni los compromisos adquiridos por la cooperativa con sus grupos de interés en términos de sostenibilidad.

Determinadas cuestiones no son objeto de comunicación en la web, como la relativa a los procedimientos para evaluar al consejo en cuanto el desempeño económico, social y medioambiental; otro caso parecido es el de las variables sobre el grado de cumplimiento de los objetivos (2\%), el impacto de las políticas en las perspectivas a largo plazo y en el desempeño financiero de la organización (3\%), la relación entre la retribución de los miembros del consejo y las cuestiones económicas, sociales y medioambientales (3\%), y la existencia de mecanismos de queja y reclamaciones ante los incumplimientos de programas diseñados con los grupos de interés (4\%), todas ellas cuestiones con una puntuación muy baja.

\subsection{Criterios de la Responsabilidad Social}

En la tabla 2 se resumen los resultados de las frecuencias obtenidas en cada una de las cinco categorías en las que se divide el estudio, en términos generales, pero también diferenciando en función del grado de la cooperativa (primer grado frente a segundo grado o ulteriores). Cada una de estas categorías representa un criterio o una perspectiva diferente desde la que se abordan los contenidos relacionados con la Responsabilidad Social.

Tabla 2. Resultados por categorías

\begin{tabular}{|c|c|c|c|c|}
\hline & $(\%)$ & $\begin{array}{c}\text { Grado } \\
\text { Cooperativa }\end{array}$ & $(\%)$ & $\begin{array}{c}\text { Desviación } \\
\text { típica }\end{array}$ \\
\hline \multirow{2}{*}{ C1. Estrategia y análisis } & \multirow{2}{*}{$8 \%$} & $1^{\circ}$ & $3 \%$ & 1,50350 \\
\hline & & $2^{\circ}$ y ulterior & $14 \%$ & 4,04651 \\
\hline \multirow{2}{*}{ C2. Perfil Organización } & \multirow{2}{*}{$40 \%$} & $1^{\circ}$ & $34 \%$ & 6,16335 \\
\hline & & $2^{\circ}$ y ulterior & $56 \%$ & 5,92546 \\
\hline \multirow{2}{*}{ C3. Parámetros Información } & \multirow{2}{*}{$31 \%$} & $1^{\circ}$ & $20 \%$ & 1,42441 \\
\hline & & $2^{\circ}$ y ulterior & $42 \%$ & 3,13535 \\
\hline \multirow{2}{*}{ C4. Gobierno Corporativo } & \multirow{2}{*}{$7 \%$} & $1^{\circ}$ & $2 \%$ & 0,82078 \\
\hline & & $2^{\circ}$ y ulterior & $13 \%$ & 5,05062 \\
\hline \multirow{2}{*}{ C5. Grupos de interés } & \multirow{2}{*}{$9 \%$} & $1^{\circ}$ & $2 \%$ & 0,82078 \\
\hline & & $2^{\circ}$ y ulterior & $15 \%$ & 4,70908 \\
\hline
\end{tabular}

Fuente: Elaboración propia. 
El análisis de resultados por categorías de la tabla 2 ofrece unas puntuaciones bajas en todas ellas en general; las cooperativas agroalimentarias podrían estar desaprovechando las oportunidades que ofrece la web como instrumento para la transparencia en términos de RSE, a pesar de que se han analizado las cooperativas con un mayor volumen de ingresos y, por lo tanto, las que disponen de unos mayores recursos para el mantenimiento y actualización de los contenidos de sus páginas web.

Sólo la categoría C2. "Perfil de la Organización" alcanza un 40\% en el nivel de información difundida. Aunque a su vez es la categoría que presenta una mayor dispersión en sus resultados. Destacar que la categoría con el resultado más bajo es la C4. "Gobierno Corporativo", con un porcentaje del 7\%. Igualmente bajas son las puntuaciones de la categoría C1. "Estrategia" (8\%), y C5. "Grupos de Interés" (9\%). La categoría C3. "Parámetros de la Información" se sitúa en un nivel intermedio del 31\%.

Por otra parte, en todas las categorías se observan unas frecuencias mayores en la cooperativas de segundo grado y ulterior que en las de primer grado.

La significatividad de este resultado ha sido contrastada mediante la realización de una prueba no paramétrica U de Mann Whitney (análoga a la prueba paramétrica de la t-student, pero más adecuada para el tamaño de la muestra de este estudio), que concluye que hay diferencias significativas entre las frecuencias obtenidas en las cooperativas de primer grado y las de $2^{\circ}$ grado y ulterior, en las categorías 2,3 y 4, como se muestra en la tabla 3.

Tabla 3. Prueba U de Mann Whitney

\begin{tabular}{|l|r|r|r|r|r|}
\hline & \multicolumn{1}{|c|}{$\mathrm{C} 1$} & \multicolumn{1}{c|}{$\mathrm{C} 2$} & \multicolumn{1}{c|}{ C3 } & \multicolumn{1}{c|}{ C4 } & C5 \\
\hline U de Mann-Whitney & 143,500 & 101,000 & 86,000 & 120,500 & 133,500 \\
W de Wilcoxon & 353,500 & 311,000 & 296,000 & 330,500 & 343,500 \\
Z & $-1,703$ & $-2,506$ & $-3,283$ & $-2,202$ & $-1,852$ \\
Sig. asintót. (bilateral) &, 089 &, 012 &, 001 &, 028 &, 064 \\
Sig. exacta [2*(Sig. unilateral)] &, $194^{\mathrm{a}}$ &, $012^{\mathrm{a}}$ &, $003^{\mathrm{a}}$ &, $050^{\mathrm{a}}$ &, $113^{\mathrm{a}}$ \\
\hline
\end{tabular}

a. No corregidos para los empates.

Fuente: Elaboración propia.

Los mayores niveles de las cooperativas de segundo grado y ulteriores en las categorías 2 y 3 podrían ser fruto de su mentalidad y objeto social, la comercialización en común de uno o varios productos a través de un grupo en forma de cooperativa de $2^{\mathrm{a}}$ grado. Esta finalidad las predispone a unos mayores niveles de atención al cliente y a una mayor 
transparencia, utilizando para ello la web, en cierta manera. En cuanto a la categoría 4, la diferencia podría estar justificada porque su gobierno presenta otras particularidades.

Es significativo el bajo nivel de las cooperativas de primer grado, quizás provocado por su acercamiento y atención más al proveedor, al socio, su propietario, que al cliente, lo que provoca una menor atención a la utilización de la web como medio para la comunicación con sus grupos de interés.

\subsection{Relación entre la Responsabilidad Social Corporativa y las características de las cooperativas}

Finalmente, se ha tratado de correlacionar los niveles de divulgación de información, por categorías, con las características de las cooperativas en cuanto a su tamaño y a la antigüedad.

Los resultados de la tabla 4 demuestran que los niveles de divulgación de información de las cooperativas agroalimentarias analizadas sólo están correlacionados en todas las categorías con el número de socios de base. Parece lógico que los resultados obtenidos demuestren que la mayor correlación se produce entre el número de socios y los aspectos relacionados con la categoría de Gobierno Corporativo.

También la facturación presenta una correlación significativa con la categoría 2. "Perfil de la Organización". Las empresas de mayor tamaño son las que presentan unos niveles de difusión de información en este apartado más elevados.

Tabla 4. Correlaciones bivariadas entre características y dimensiones

\begin{tabular}{|l|c|c|c|c|}
\hline & $\begin{array}{c}\text { Facturación } \\
(\mathrm{M} €)\end{array}$ & $\begin{array}{c}\mathrm{N}^{\circ} \text { empleos } \\
\text { directos }\end{array}$ & $\begin{array}{c}\mathrm{N}^{\circ} \text { de socios } \\
\text { de base }\end{array}$ & Antigüedad \\
\hline C1. Estrategia & $\begin{array}{c}\text { No } \\
\text { significativa }\end{array}$ & $\begin{array}{c}\text { No } \\
\text { significativa }\end{array}$ & 0,441 & $\begin{array}{c}\text { No } \\
\text { significativa }\end{array}$ \\
\hline C2. Perfil Organización & 0,403 & $\begin{array}{c}\text { No } \\
\text { significativa }\end{array}$ & 0,415 & $\begin{array}{c}\text { No } \\
\text { significativa }\end{array}$ \\
\hline C3. Información & $\begin{array}{c}\text { No } \\
\text { significativa }\end{array}$ & $\begin{array}{c}\text { No } \\
\text { significativa }\end{array}$ & 0,480 & $\begin{array}{c}\text { No } \\
\text { significativa }\end{array}$ \\
\hline C4. Gobierno Corporativo & $\begin{array}{c}\text { No } \\
\text { significativa }\end{array}$ & $\begin{array}{c}\text { No } \\
\text { significativa }\end{array}$ & 0,630 & $\begin{array}{c}\text { No } \\
\text { significativa }\end{array}$ \\
\hline C5. Grupos de interés & $\begin{array}{c}\text { No } \\
\text { significativa }\end{array}$ & $\begin{array}{c}\text { No } \\
\text { significativa }\end{array}$ & 0,500 & $\begin{array}{c}\text { No } \\
\text { significativa }\end{array}$ \\
\hline
\end{tabular}

Fuente: Elaboración propia. 
En cualquier caso, las correlaciones significativas obtenidas no son determinantes en la mayoría de los casos (escasamente alcanzan el 50\%) y presentan unos valores poco relevantes como para poder concluir que el tamaño o la antigüedad de la cooperativa sean aspectos determinantes en los niveles de difusión de información.

\section{CONCLUSIONES}

En este trabajo se han analizado algunos aspectos sobre la transparencia en materia de responsabilidad social de la empresa cooperativa agroalimentaria española y, en particular, los niveles de información sobre RSE divulgados a través de las páginas webs, agrupados en diferentes categorías.

El estudio ha constatado la existencia de unos niveles bajos en la difusión de este tipo de información en la mayoría de las empresas cooperativas agroalimentarias analizadas ya que éstas utilizan su web fundamentalmente con fines comerciales. Estos resultados están en consonancia con los de otros estudios recientes como los de López y otros (2012), y Montegut y otros (2013) que demuestran un uso de la web con fines transaccionales más que de carácter relacional. En general, la información sobre RSE es escasa, aparece de manera dispersa y poco sistemática, y no hay uniformidad en los contenidos. La web no se configura como un medio de comunicación y diálogo con los grupos de interés de cara a mejorar la transparencia, sino como plataforma para la venta de sus productos.

Los contenidos sobre el perfil de la organización destacan sobre otras categorías esenciales en la gestión de la RSE como la relativa al gobierno corporativo o las relaciones con los grupos de interés que presentan unos niveles de divulgación insuficientes. Se puede concluir que las cooperativas están desaprovechando las oportunidades que ofrece internet como medio para mejorar la transparencia y la rendición de cuentas.

En general, no se puede confirmar de manera concluyente que los valores implícitos en los principios cooperativos o los resultados positivos de sus políticas de RSE se hayan traducido de una manera determinante en unos niveles de transparencia importantes, aunque sí se puede apreciar de manera clara que las cooperativas de segundo grado y ulteriores presentan unos niveles muy superiores a las de primer grado en cuanto a la difusión de información. 
En general, en la información que se difunde a través de la web priman las variables relacionadas con la identificación del negocio y con los reconocimientos (marcas, premios, mercados, localización, forma jurídica) más que con la forma cómo se han articulado las políticas de RSE (iniciativas, canales de comunicación, procedimientos para la evaluación y medición de impactos) o con los compromisos adquiridos en materia de sostenibilidad con los stakeholders.

En cuanto a los posibles factores explicativos de los mayores niveles de difusión de información, sólo el número de socios parece condicionar unos mayores esfuerzos de transparencia en términos de RSE. Ni la facturación ni el número de empleados parecen ser determinantes. Tampoco la antigüedad de las cooperativas aunque a priori los años de vida pudieran ser un síntoma de la madurez con la que se abordan cuestiones tan íntimamente relacionadas con los valores cooperativos, como las relativas a su responsabilidad social.

Finalmente, cabría decir que el estudio podría estar limitado por la definición de variables que realiza la propuesta GRI, en la que se ha basado su selección, y que podría no estar adaptada a las necesidades de información del sector cooperativo, a pesar de haber considerado en este estudio la adaptación sectorial a las entidades sin ánimo de lucro. En futuras investigaciones cabría la posibilidad de analizar los niveles de difusión de información utilizando las variables incorporadas en otras iniciativas, como los códigos de conducta, y a través de otros canales de comunicación diferentes a la web.

\section{BIBLIOGRAFÍA}

ACCOUNTABILITY (2008a) AA1000 AccountAbility Principles Standard, London, Accountability.

ACCOUNTABILITY (2008b) AA1000 Assurance Standard, London, Accountability.

ARCAS LARIO, N. (DIR.) (2011) El Gobierno de las cooperativas agroalimentarias. Factores de Éxito, Fundación Cajamar, Almería, 101 p.

ARCAS LARIO, N. y BRIONES PEÑALVER, A. J. (2009) Responsabilidad Social Empresarial de las Organizaciones de Economía Social. Valoración de la misma en las empresas de la Región de Murcia, CIRIEC-España, Revista de Economía Pública, Social y Cooperativa, no 65, pp. 143-165. 
BANCO MUNDIAL (2007) Environmental, Health, and Safety (EHS) Guidelines, (Washington, DC: Environment and Social Development Department, International Finance Corporation).

CARRASCO, I. (2005) La Ética como Eficiencia: la Responsabilidad Social en las Cooperativas de Crédito Españolas, CIRIEC-España, Revista de Economía Pública, Social y Cooperativa, $\mathrm{n}^{\circ}$ 53, pp. 351-367.

CASTILla POLO, F. y GALLARDO VÁZQUEZ, D. (2011) Fondo y Forma de la Revelación Social en Sociedades Cooperativas, comunicación presentada en el XVI Congreso AECA, Granada, 21-23 septiembre.

COMITÉ ECONÓMICO Y SOCIAL EUROPEO (2012) Dictamen sobre Cooperativas y desarrollo agroalimentario, NAT/541.

DEGLI, G. y PORTALE, E. (2011) The Effect of Corporate Social Responsibility on Social Capital Creation in Social Cooperatives, Nonprofit and Voluntary Sector Quarterly, vol. $40, \mathrm{n}^{\mathrm{o}} 3$, pp. 566-582.

FERRER, A.; ONTALBA, J. A. y PESET, F. (2007) El uso de internet en las empresas Cooperativas Valencianas, Economía Social, n 38, pp. 30-34.

GLOBAL REPORTING INITIATIVE (2011a) Guía para la elaboración de Memorias de Sostenibilidad versión 3.1. Disponible en internet: www.globalreporting.org/resourcelibrary/Spanish-G3.1-Reporting-Guidlines.pdf .

GLOBAL REPORTING INITIATIVE (2011b) Sustainability Reporting Guidelines and NGO Sector Supplement. Disponible en internet: www.globalreporting.org/resourcelibrary/NGOSS-Complete.pdf .

GLOBAL REPORTING INITIATIVE (2011c) Sustainability Reporting Guidelines and Food Processing Sector Supplement. Disponible en internet: Www.globalreporting.org/resourcelibrary/FPSS-Complete.pdf.

HORRACH, P. y SOCIAS, A. (2011) La Actitud de las Empresas de Economía Solidaria frente a la Divulgación de Información sobre Sostenibilidad desde el prisma de la Teoría de los Stakeholders o Grupos de Interés, Revista de Contabilidad-Spanish Accounting Review, vol. 14, nº extraordinario, pp. 267-297.

JULIÁ, J. F.; GARCÍA, G. y POLO, F. (2004) La Información Divulgada a través de internet por las Cooperativas, CIRIEC-España, Revista de Economía Pública, Social y Cooperativa, no 49, pp. 167-192. 
LÓPEZ BECERRA, E. I.; ARCAS LARIO, N. y ALCÓN PROVENCIO, F. (2012) Los sitios web de las Cooperativas Agroalimentarias. Antecedentes y consecuencias de su adopción, CIRIEC-España, Revista de Economía Pública, Social y Cooperativa, nº 76, pp. 261-282.

MONTEGUT SALLA, Y.; CRISTOBAL FRANSI, E. y GÓMEZ ADILLÓN, M. J. (2013) La Implementación de las TIC en la gestión de las Cooperativas Agroalimentarias: el caso de la provincia de Lleida, REVESCO, Revista de Estudios Cooperativos, n ${ }^{\circ} 110$, pp. 223253.

MORALES GUTIÉRREZ, A. C. (2005) Círculos Virtuosos en la Cooperación, Sociedad Cooperativa, abril, $\mathrm{n}^{\circ} 15$, pp. 30-34.

MORALES GUTIÉRREZ, A. C. y ARIZA MONTES, J. A. (2010) Sistemas de Gobierno Corporativo en las Empresas Propiedad de los Trabajadores, Revista de la Responsabilidad Social de la Empresa, n 5, Revista electrónica de la Fundación Luis Vives. Disponible en internet: www.fundaciónluisvives.org/rse/digital/5/artículos/53946/index.html.

MOZAS MORAL, A. y PUENTES POYATOS, R. (2010) La Responsabilidad Social Corporativa y su paralelismo con las Sociedades Cooperativas, REVESCO, Revista de Estudios Cooperativos, $\mathrm{n}^{\circ}$ 103, pp. 75-100.

MOZAS MORAL, A., PUENTES POYATOS, R. y BERNAL JURADO, R. (2010) La Ecorporate Social Responsibility en las sociedades Cooperativas Agrarias Andaluzas, CIRIEC-España, Revista de Economía Pública, Social y Cooperativa, $\mathrm{n}^{\circ}$ 67, octubre, pp. 187-212.

MUÑOZ PASADAS, R.; TIRADO VALENCIA, P. y RUIZ LOZANO, M. (2010) Una propuesta de herramientas para la gestión de la transparencia en las entidades no lucrativas, Revista de Fomento Social, nº 260, pp. 695-726.

NACIONES UNIDAS. GLOBAL COMPACT (UNGC) (2009) What is the Global Compact?.

Disponible en internet: www.unglobalcompact.org.

OBSERVATORIO SOCIOECONÓMICO DEL COOPERATIVISMO ESPAÑOL (2011) Informe del OSCAE. Disponible en internet: $\underline{\text { www.agro- }}$ alimentarias.coop/ficheros/doc/03723.pdf .

OCDE Organization for Economic Co-operation and Development (2006) Intellectual Assets and Value Creation: Implications for Corporate Reporting, Paris: Corporate Affairs Division, Directorate for Financial and Enterprise Affairs, OECD.

OLMEDO CIFUENTES, I; MARTÍNEZ LEÓN, I. M.; ARCAS LARIO, N. y LONGINOS MARÍN RIVES, J. (2012) Relación Circular entre Ética, Responsabilidad Social y 
Reputación de las Cooperativas, REVESCO, Revista de Estudios Cooperativos, $\mathrm{n}^{\circ}$ 107, pp. 129-154.

PALOMO ZURDO, R. J. y VALOR MARTÍNEZ, C. (2004) El “activismo" de los socios como catalizador de la Responsabilidad Social de la Empresa: aplicación a Organizaciones de Participación, CIRIEC-España, Revista de Economía Pública, Social y Cooperativa, $\mathrm{n}^{\circ}$ 50, pp. 167-190.

PEDROSA ORTEGA, C. y GARCÍA MARTÍ, E. (2011) El Gobierno en la Sociedad Cooperativa como Base de la Cohesión Social: el caso de una cooperativa agraria, REVESCO, Revista de Estudios Cooperativos, $\mathrm{n}^{\circ} 106$, pp. 7-32.

PÉREZ SANZ, F. J. y GARGALLO CASTEL, A. (2005) Gestión, Desarrollo y Aplicación de la Responsabilidad Social en Entidades de Economía Social: el caso de las cooperativas, CIRIEC-España, Revista de Economía Pública, Social y Cooperativa, nº 53, noviembre, pp. 305-332.

PÉREZ SANZ, F. J., ESTEBAN SALVADOR, L. y GARGALLO CASTEL, A. (2009) Participación, Gobierno Democrático y Resultados Cooperativos: una perspectiva de RSE, CIRIEC-España, Revista de Economía Pública, Social y Cooperativa, no 65, agosto, pp. 163-190.

PUENTES POYATOS, R., VELASCO GÁMEZ, M. M. y VILAR HERNÁNDEZ, J. (2009) El Buen Gobierno Corporativo en las Sociedades Cooperativas, REVESCO, Revista de Estudios Cooperativos, ${ }^{\circ}$ 98, pp. 118-140.

REY GARCÍA, M. y MARTÍN CABANA, J. (2011) Buen Gobierno y Rendición de Cuentas en las Fundaciones Empresariales Españolas: un análisis comparativo de prácticas de Transparencia, Revista de la Responsabilidad Social de la Empresa, $\mathrm{n}^{\circ}$ 7, Revista electrónica de la Fundación Luis Vives. Disponible en internet: www.fundaciónluisvives.org/rse/digital/7/artículos/60190/index.html .

SALAZAR, L. (2010) ¿Cómo Funciona el Gobierno Cooperativo en las Cooperativas de Villavicencio?, Revista Cooperativismo y Desarrollo, vol. 18, $\mathrm{n}^{\circ}$ 97, julio-diciembre, pp.126-138.

SERVER IZQUIERDO, R. y CAPÓ VICEDO, J. (2009) La Responsabilidad Social Empresarial en un contexto de crisis. Repercusión en las Sociedades Cooperativas, CIRIEC-España, Revista de Economía Pública, Social y Cooperativa, no 65, agosto, pp. 731. 